\title{
Note sul sintagma aggettivale in italiano antico
}

\section{Some Remarks on Adjective Phrase in Old Italian}

Francesco Bianco [francesco.bianco@upol.cz]

Univerzita Palackého v Olomouci, Repubblica Ceca

Benedetto Giuseppe Russo [benedetto.russo@tiscali.it]

Univerzita Palackého v Olomouci, Repubblica Ceca

\section{Riassunto}

Il presente contributo analizza le funzioni del sintagma aggettivale nelle varietà italoromanze medievali. La parte centrale dell'articolo $(\$ 2)$ è dedicata all'aggettivo qualificativo, quale prototipo del sintagma aggettivale: particolare attenzione è dedicata al suo ruolo $(\$ 2.2)$ e alla sua posizione $(\$ 2.3)$ nella frase. Anche la struttura argomentale dell'aggettivo è considerata $(\$ 2.4)$. Il $\$ 3$ è dedicato agli aggettivi pronominali: oltre a classi ben conosciute (per es. i possessivi), si considera anche la meno nota funzione aggettivale del relativo ( $\$ 3.4)$. L'ultimo paragrafo $(\$ 4)$ getta una luce su costrutti di diverso tipo (sintagmi preposizionali e frasi relative) ma che condividono alcuni caratteri degli aggettivi propriamente detti. Gli esempi sono tratti da testi di generi differenti: prosa letteraria, poesia, testi pratici e soprattutto la cosiddetta "prosa media", tipica del Medio Evo italoromanzo.

\section{Parole Chiave}

Aggettivo; italiano antico; sintagma aggettivale; sintassi

\begin{abstract}
This paper deals with the functions of the adjective phrase in Italo-Romance language varieties in Middle Ages. The central section of the paper $(\$ 2)$ is dedicated to qualifiers, as prototype of adjective phrase: their role $(\$ 2.2)$ and their position $(\$ 2.3)$ within the sentence are expecially focused. A section is also dedicated to their argument structure $(\$ 2.4)$. The third section $(\$ 3)$ is dedicated to such adjectives, which come from pronouns: other than some well known classes (e.g. possessive adjectives), the quite unfamiliar adjective role of the relative prononun is also investigated $(\$ 3.4)$. Finally, the $₫ 4$ aims to shed light on some different constructs, such as prepositional phrases and relative clauses, which share some functions and features of the adjectives. The examples are taken from several genres: literary prose, poetry, practical texts, and above all the so-called "prosa media", typical of the Italian Middle Age.
\end{abstract}




\section{KEYWORDS}

Adjective; Adjective phrase; Old Italian; Syntax

RICEVUTO 2020-02-17; ACCETTATO 2020-06-08

Il presente contributo è stato sviluppato in seno all'Archivio della Sintassi dell'Italiano Letterario (ArSIL), progetto nato nel 2001 e coordinato da Maurizio Dardano (cfr. Dardano 2009 e SIA: IX-XI, 1-35).

A Francesco Bianco si devono i $\$ \$ 2.3,3$ e 4, a Benedetto Giuseppe Russo il $\$ 2$ (ad eccezione del $\$ 2.3$ ). L'introduzione $(\$ 1)$ e le conclusioni $(\$ 5)$, così come l'ideazione e la revisione dell'articolo, sono da attribuirsi a entrambi gli autori.

La realizzazione e la pubblicazione del presente dellarticolo sono state possibili grazie al finanziamento per la ricerca universitaria erogato all’Università Palacký di Olomouc dal Ministero della pubblica istruzione, della gioventù e dello sport della Repubblica Ceca; esse si inscrivono nel quadro del progetto IGA_FF_2020_023 (Nuove linee di ricerca nei paesi di lingua romanza: letteratura, linguistica e cultura).

\section{Introduzione}

Secondo la definizione corrente, il sintagma aggettivale [d’ora in poi SA] «è un sintagma endocentrico che ha un aggettivo come testa» (EncIt: 1352, s.v. Tipi di sintagma). In base a tale definizione, è dunque necessario che il SA contenga un aggettivo; sono esclusi altri costrutti, quali le frase relative $[\mathrm{FR}]$ e i sintagmi preposizionali [SP], che pur condividono la funzione principale del SA: quella di modificare un sintagma nominale [SN].

La medesima distinzione è presente già nella GGIC, che dedica sezioni diverse dellopera al SA (II, 321-337) e al SP (I, 521-545); la sezione sul SN (I, 287-517), tuttavia, ospita sia il capitolo sulla FR (I, 457-517) sia un (altro) capitolo sul SA (I, 439-455). In effetti, sotto il profilo funzionale, non sembrano esserci differenze fra (1), (2) e (3):

1) Mario è una persona ricca

2) Mario è una persona con molto denaro

3) Mario è una persona che possiede molto denaro

Anche quando svolge funzione predicativa, un SA (4) può essere sostituito da un SP (5); in questo caso, tuttavia, non è possibile ricorrere a una FR, come mostra l'es. (6):

4) Questo ristorante è molto buono

5) Questo ristorante è di grande qualità

6) ${ }^{\star}$ Questo ristorante è che è molto buono/che è di grande grande qualità

Nel presente contributo, in ossequio alla definizione tradizionale, accorderemo all'aggettivo il ruolo di "prototipo" del SA, focalizzandoci sulle sue forme, sulle sue funzioni e sui suoi usi in 
italiano antico ${ }^{1}$. Ci concentreremo, in primis, sugli aggettivi qualificativi, quindi sugli aggettivi pronominali; concluderanno il lavoro, infine, alcuni rapidissimi confronti con costrutti di altro tipo - SP e FR -, che ci permetteranno di mettere in luce differenze e affinità di funzione e comportamento.

\section{Aggettivi qualificativi}

L'aggettivo qualificativo esprime una qualità (Dardano, Trifone 1997: 197) o modo di essere del referente indicato dal nome $[\mathrm{N}]$ cui è legato semanticamente e grammaticalmente: in base, cioè, alle norme sintattiche e, in gran parte dei casi, attraverso l'accordo morfologico (Serianni 2010[1989]: 191). È infatti una parte variabile del discorso, soggetta a flessione grammaticale nel genere e nel numero (limpido/limpida; limpidi/limpide; ipocrita/ipocriti, ipocrite) o solo nel numero (forte/forti; pregevole/pregevoli), sebbene esista anche una classe di aggettivi invariabili: pari, rosa, arrosto, ecc.

A differenza dei pronominali, gli aggettivi qualificativi costituiscono una classe di parole aperta, in quanto soggetta all'arricchimento lessicale, motivato dalla necessità di designare idee, qualità, forme, ecc. con parole nuove e condizionato dai cambiamenti della società e della cultura, ma anche da mutamenti semantici consistenti nellattribuzione a vocaboli già esistenti di accezioni o sfumature di significato diverse da quelle del passato (Serianni 2010[1989]: 192; Dardano, Trifone 1997: 198). In un dinamismo aperto tanto verso l'addizione quanto verso la perdita, molti aggettivi subiscono altresì una progressiva decadenza nell'uso fino a divenire forme arcaiche obsolete, ovvero preziosismi confinati nell'uso letterario e poetico o suscettibili di ripescaggi ironici e comici².

\subsection{Aggettivi di relazione ${ }^{3}$}

Gli aggettivi di relazione costituiscono una classe peculiare di qualificativi: si tratta di aggettivi denominali che, piuttosto che indicare una proprietà del nome cui si riferiscono, mettono tale nome in relazione con quello da cui derivano e del quale traspongono il contenuto semantico nella categoria aggettivale. Non esprimono dunque qualità ma rimandano all’entità designata dal

1 In questa sede si accoglie una nozione piuttosto ampia di "italiano antico", che incorpora testi italoromanzi di qualsiasi provenienza geografica, redatti in un arco cronologico che va dalle Origini alla fine del XIV sec. I pochi sconfinamenti sono serviti, per lo più, a scopo di confronto. Per fornire un quadro il più possibile completo e affidabile, si è scelto di considerare sia documenti letterari sia documenti non letterari, appartenenti a diversi generi testuali, fra cui abbondano quelli ascrivibili alla cosiddetta "prosa media» (Dardano 1969: 9-10). Coerentemente con le scelte soggiacenti al progetto $A r S I L$ (cfr. p. 110, nota iniziale), per le quali si rimanda al $\$ 1$ della SIA (1-35), si è scelto di includere anche i volgarizzamenti; parimenti, pur riconoscendo che «[n]el Medioevo la prosa e la poesia percorrono cammini tra loro distinti, anche per quanto riguarda le scelte sintattiche» (ivi: 1), abbiamo ritenuto opportuno non escludere del tutto i testi in versi, il ricorso ai quali è stato sempre prudente e valutato caso per caso.

2 Si possono ricordare, con Coletti (2018: 37-38; 45-49): algente 'gelato', bambo 'sciocco', callido 'astuto', corrusco 'splendente', imo 'che si trova in fondo', inope 'povero', lippo 'cisposo', parvo 'piccolo', perso 'bruno rossiccio', pravo 'malvagio', ratto 'rapido', venusto 'bello'.

3 Gli aggettivi di relazione sono chiamati classificatori da Giusti (GIA: 601, 605; cfr. anche Brinker 1974, Serianni 2010[1989]:192-194, Dardano, Trifone 1997: 197-198 e 229-230, Russo 2009, EncIt: 29-32, s.v. Aggettivi; in prospettiva romanza Bortolotto 2016). 
nome cui sono legati morfologicamente e da una stabile relazione semantica (nominale $\leftarrow$ nome; annuale $\leftarrow$ anno $)$.

Brinker (1974: 7) attribuisce all'aggettivo di relazione la funzione di trasporre in forma aggettivale un SP, solitamente di $+\mathrm{N}$ (luce del sole $\rightarrow$ luce solare); a livello semantico e anche formale, tuttavia, tale trasposizione non sempre è applicabile.

Tra gli aggettivi di relazione si possono annoverare gli etnici, indicanti l'appartenenza a un'area geografica, una nazione, una regione, una città, un paese, come il già citato egiziano, africano, calabrese, siciliano, milanese, pisano (Marcato 2010), e quelli derivati da antroponimi, come ciceroniano, cristiano, petrarchesco. Nell'italiano antico come in quello moderno esistono, inoltre, aggettivi relazionali derivati da basi suppletive, ossia da radici di origine dotta (latina o greca); il rapporto morfologico tra il nome base nella forma corrente e il derivato aggettivale è, in

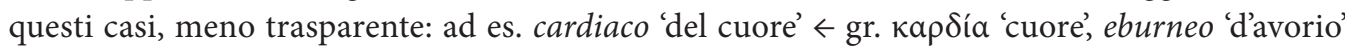

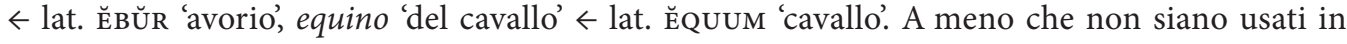
un'accez. qualificativa / figurata, tali aggettivi non ammettono gradazioni in senso comparativo e superlativo né possono essere modificati da avverbi intensificatori ( ${ }^{\star}$ più / meno angolare; ${ }^{*}$ metallicissimo; ${ }^{*}$ molto geografico).

Laggettivo di relazione ha sempre un valore intrinsecamente restrittivo, cioè limita il campo di riferimento ad alcuni degli oggetti, concetti o esseri designati dal nome cui si riferisce e permette di distinguere questi ultimi da altri della stessa categoria: ha pertanto una funzione identificativa e offre un'informazione indispensabile per individuare il referente espresso dal SN di cui fa parte. Quanto alla collocazione all'interno di quest'ultimo, come i qualificativi con valenza restrittiva e in quanto finalizzato a stabilire un legame tra il nome che modifica e quello da cui proviene (la sua base), in italiano moderno l'aggettivo di relazione con significato proprio (non qualificativo, dunque) si trova dopo il nome cui si riferisce (rapporti sessuali, il sapere scientifico; le sequenze ?sessuali rapporti, ?lo scientifico sapere sono possibili solo in contesti marcati in senso letterario). In italiano antico, invece, esso è postnominale nellordine non marcato, cosa che vale anche per gli aggettivi «di nazione» (GIA: 605), ossia gli etnici (7), ma è possibile e frequente anche la posizione prenominale. Ciò avviene tanto in poesia (8), quanto in prosa; gli ess. (9)-(10) mostrano l'alternanza posizionale di femminile:

7) Mercatanti fiorentini passavano in nave per andare oltramare (Rettorica, p. 109);

8) Cesare, poi chebbe, per tradimento / dell'egizian duttor, l'orrate chiome, / rallegrossi nel core, en vista come / si fa qual che di nuovo è discontento (Boccaccio Rime, I, XLI, 2, p. 41);

9) Femminile cosa è liticare e contendere e mostrare l'ira sua in costumi (Fiori, XXIV, p. 183);

10) Adam [...] cadde nella disobbedienzia, e dalla disobbedienzia alla immondizia, con superbia $e$ piacere feminile (C. da Siena Libro, CXXXV, p. 304).

\subsection{Funzioni dell'aggettivo qualificativo in italiano antico}

Laggettivo qualificativo svolge due fondamentali funzioni: attributiva e predicativa; a queste ne va aggiunta una terza, quella avverbiale, presente anche nell'italiano antico, che prevede però la conversione ad altra categoria grammaticale. 
Esso svolge funzione attributiva quando modifica semanticamente un nome dal quale dipende e che, a sua volta, può avere funzioni diverse; gli ess. che seguono presentano attributi del soggetto (11), di un complemento indiretto (12), di un nome con funzione predicativa, retto da essere (13) o da altro verbo copulativo (14), di un'apposizione (15):

11) le giovani donzelle sono di poca stabilità e per la loro bellezza da molti amanti sogliono essere stimolate (Filocolo, III, XIII, p. 261);

12) questo nome Ercole è soprannome delli uomini molto forti (Guido da Pisa, CIX, p. 218);

13) Però vedete omai quanto s'acquista / studiando l'alta fantasia profonda / de la qual Dante fu comico artista! (Capitolo, CXLIV, p. 369);

14) montò tra lloro tanta invidia che divennero nemici mortali (Rettorica, p. 94);

15) Ma i fratelli, animosa schiera, e accesa di lutto, parte prendono le coltella, parte prendono saettamenti, e ciechi ruinano (Ciampolo, XII, p. 407).

Laggettivo ha funzione predicativa quando è retto direttamente dal verbo essere o da un altro verbo copulativo, costituendo, rispettivamente il nome del predicato (16) o il complemento predicativo del soggetto (17); ha, invece, la funzione di complemento predicativo dell'oggetto quando il verbo copulativo al quale è legato è transitivo e regge un oggetto diretto, al quale appunto l'aggettivo si riferisce (18):

16) temperata quantità di queste cose basta all'uomo acciò che egli sia felice (Tesoro Gaiter, vol. 3 , VI, LVI, p. 175);

17) in verità lo ingengno diventa infermo se elli non è aitato (et) riparato co(n) cotidiano legere (Trattati Albertano, Liber cons. (pisano), IX);

18) Ma questa generazione di combattere non giudicano ottima coloro che sono d'arme scaltriti (Vegezio, III, XX, p. 126).

Se l'aggettivo è unito a un verbo predicativo alla cui predicazione aggiunge la propria, esso è portatore di una predicazione secondaria ${ }^{4}$, che può riguardare il soggetto o l'oggetto diretto, come si vede, rispettivamente, in (19) e (20):

19) Quando l'aira rischiara e rinserena, / il mondo torna in grande diletanza / e l'agua surge chiara de la vena (Bondie Dietaiuti, II, 3, p. 143);

20) La natura crea gli uomini forti per animo, ma 'l savere gli redde migliori per buoni ammaestramenti (Vegezio, III, XXVI, p. 143).

Gli aggettivi con funzione attributiva possono avere valore appositivo (o descrittivo) e valore restrittivo. Nel primo caso, l'aggettivo esprime una qualità intrinseca del referente indicato dal nome o soggettivamente attribuita a tale referente dal parlante, e si limita ad arricchire il nome di elementi caratterizzanti ma non necessari per la comprensione del suo significato fondamentale (un vecchio mobile; una dolce bambina). Nel secondo, come si è anticipato trattando degli

4 Cfr. Salvi (1981), GGIC (II, 191-226), GIA (191-239), EncIt (1136-1139, s.v. Complemento predicativo). 
aggettivi di relazione, l'attributo reca un'informazione indispensabile, identificativa, che circoscrive il riferimento del nome, limitando la portata referenziale del SN di cui fa parte (una casa nuova; una famiglia ricca). Come in italiano moderno, in italiano antico l'aggettivo appositivo solitamente precede il nome:

21) alcuna volta ramorbidava colle dita la bionda cera (Simintendi Volg. Metamorfosi, vol. 2, VIII, p. 135).

Si registrano invece differenze nella posizione dell'aggettivo restrittivo: in italiano moderno è ammessa solo la posizione postnominale; in italiano antico si ha anche la posizione prenominale, come mostra l'es. (22); per la posizione postnominale, cfr. (23) e il precedente (12); si noti come violento, aggettivo presente in (22) e (23), abbia funzione restrittiva in entrambe le posizioni:

22) O gente cieca e non saputa di crudele morte, perchè non con violenta mano levate via Elena dal non giusto marito e lei vi studiate di renderla al giusto marito [...]? (Mazzeo, p. 457);

23) $\mathrm{P}(\mathrm{er})$ ché queste parte sono carnacciose et no(n) sono piene di vene, arterie et nervi, sicuramente vi possiamo tagliare, incendere, et ponere unguenti violenti (Maestro Bartolomeo Chirurgia, IV, XIV, p. 293).

All'interno di un SN, due aggettivi coordinati possono trovarsi sia prima $(24,27)$ sia dopo il nome (25-26), con valore tanto appositivo (24-25) quanto restrittivo (26-27); possono altresì trovarsi uno prima e uno dopo il nome (28); possibilità, questa, preclusa all'italiano moderno (se non in formule cristallizzate):

24) Ma pur egli si riconforta vedendo il chiaro e bello viso della reina Ginevra (TR, p. 340);

25) Questa battaglia grande e famosa fue appo Azio (Orosio, VI, XVIII, p. 409);

26) Del mese di Settembre si tolgano gli alveari vecchi e gravi, li quali nella passata State non composono sciami (Crescenzi, vol. 3, IX, CIV, p. 166);

27) E non ti dei pensare orribile cosa se 'l picciolo regnio nel quale in qua dirieto se' stata tu commuti in ampii e grandi regnami, perciò che al reame di Troia sono molti reami sotto posti (Mazzeo, p. 455);

28) e egli entrò in fiera malinconia e ispiacevole (Dec, III, 7, 5, p. 590).

\subsection{Topologia dell'aggettivo qualificativo}

Un aggettivo può essere accompagnato da un modificatore, come un avverbio, e/o da un complemento retto da una preposizione, al posto del quale si può trovare una proposizione con modo finito o indefinito. L'italiano antico presenta un ordine delle parole all'interno del SA relativamente libero rispetto all'italiano moderno, con cui condivide l'ordine non marcato. Un modificatore, come gli avverbi di quantità molto e assai, può trovarsi a sinistra dell'aggettivo, come in italiano moderno (29), così come a destra di quest'ultimo (30), possibilità che la lingua attuale esclude: 
29) Et è questo modo della tentatione molto sottile et periculoso, però chè aitata quella tentatione dalla natura (Genesi, VIII, p. 90);

30) Era Pericone uomo di fiera vista e robusto molto (Dec II, 7, 22, p. 403).

Lordine non marcato aggettivo-complemento coesiste con quello marcato complemento-aggettivo; si osservi, in (31), il chiasmo prodotto dalla compresenza di entrambi gli ordini:

31) E uscite dell'acque e di mortine coronate, in uno grazioso seno, che 'l monte di sé faceva quivi vicino, di bellissima erba pieno e dipinto di molti fiori, ce ne andammo (Ninfe, XXXII, 55, p. 780).

Quando l'aggettivo è accompagnato sia da un modificatore sia da un complemento, oltre all'ordine non marcato (32), possiamo trovare sequenze diverse (33-35):

32) e però che lla natura di quelli tiranni è molto conforme a' tradimenti [...] (Giovanni Villani, I, IV, p. 548);

33) fue uomo di grosso intelletto quanto ne la pratica cortigiana, ma sofficiente assai in iscrittura (Giovanni Villani, III, XII, p. 65);

34) sopra l'altro canto il palido ulivo, caro a Pallade molto, di rami pieno si vedea e di frondi (Ninfe, XXVI, 33, p. 748);

35) E se quel giorno fu a te molto glorioso e al Comune molto piacevole, non t'incresca, Iulio Cesare, per Dio, d'acquistare sovente lode di così dilettosa gloria (Pro Ligario, p. 184).

Contigui in italiano moderno, in italiano antico gli elementi del SA possono anche trovarsi, invece, in posizione discontinua, cioè separati da elementi nominali o verbali a esso esterni, con collocazione periferica del modificatore a inizio proposizione (36) o dopo il nome preceduto dall'aggettivo (37):

36) avegna che molto fosse orrevole e ricca, molti naveva veduti e provati (Nov, LXXXVI, 2, p. 145);

37) Ella, la quale era formosa e di piacevole aspetto molto, [...] a Neifile comandò che alle future novelle con una desse principio (Dec, II, Introduzione, 4, p. 311).

Anche il complemento può non essere contiguo all'aggettivo da cui dipende (38). Similmente, una proposizione limitativa con il verbo all'infinito può essere contigua all'aggettivo (39) o seguire il sostantivo con cui l'aggettivo concorda (40):

38) Sopra che ciascun pensi di dire alcuna cosa che alla brigata esser possa utile o almeno dilettevole (Dec, II, Conclusione, 9, p. 493);

39) cosa nova a vederla, / già santissima et dolce anchor acerba, / parea chiusa in òr fin candida perla (RVF, CCCXXV, 78-80, p. 1264);

40) propuosi di dire parole di questo che mera adivenuto, però che mi parea che fosse amorosa cosa da udire (VN, XIV, 16, p. 131). 
Quanto alla collocazione del SA complesso rispetto al SN cui si riferisce, Giusti (GIA: 597) ha individuato le condizioni in presenza delle quali tale sintagma può comparire in posizione prenominale: a) l'aggettivo non è accompagnato da un complemento ma solo da un eventuale modificatore (41); b) quest'ultimo precede l'aggettivo (42); c) il SN cui il SA è legato non contiene l'articolo determinativo (43):

41) Democrito fue molto grande filosofo (Fiori, II, p. 106);

42) aveavi una molto bella donna la quale era molto sguardata dalle genti (Nov, XXVI, 2, p. 51);

43) mi fu egli di grandissima fatica a sofferire, certo non per crudeltà della donna amata, ma per soverchio fuoco nella mente concetto da poco regolato appetito (Dec, Proemio, 3, p. 128).

Figurano generalmente prima del nome, nellordine non marcato, gli aggettivi di dimensione (44) e quelli che esprimono valutazione (45), per lo più generica:

44) un altro filosafo [...] venne a lui e trovò grandi letta ne la camera sua (Fiori, VIII, p. 123);

45) Menârlo in una bella cittade; cavalieri li mostraro di gran paraggio, bel destriere e bell'arme li apprestaro (Nov, XXI, 16, p. 44).

In posizione prenominale troviamo anche aggettivi con significato comparativo, come simigliante (46); comparativi (47) e superlativi (48) organici; aggettivi di grado comparativo e superlativo (49-50):

46) Giannotto non stette per questo che egli, passati alquanti dì, non gli rimovesse simiglianti parole (Dec, I, 2, 8, p. 221);

47) Maggiore disinore è perdere o male spendere quello che l'uomo ha guadagnato, che non sarebbe non avere guadagnato (Fiori, XXI, p. 170);

48) in queste due cose si sente quel piacere altissimo di beatitudine lo quale è massimo bene in Paradiso (Cv, III, XV, p. 243);

49) Tancredi, serbati coteste lagrime a meno disiderata fortuna che questa ( $D e c, I V, 1,60, p .713$ );

50) questi nonn è servo, ma malaventurato servo, poscia che sia nato di gentilissima schiatta (Fiori, XX, p. 164).

Quando il nome è accompagnato da un suo complemento e da un aggettivo, si possono trovare ordini diversi:

51) E tratti del paniere oricanni d'ariento bellissimi e pieni qual d'acqua rosa, qual d'acqua di fior d'aranci, qual d'acqua di fiori di gelsomino e qual d'acqua nanfa, tutti costoro di queste acque spruzzarono (Dec, VIII, 10, 18, p. 1338);

52) essendo Guido partito d'Orto San Michele e venutosene per lo Corso degli Adimari infino a San Giovanni [...], essendo arche grandi di marmo [...] (Dec, VI, 9, 10, pp. 1020-1021). 
Quando è usato con funzione intensificatrice, bello precede il sostantivo e il suo complemento (53), come in italiano moderno ${ }^{5}$. Possibili, sebbene assai rari, i controesempi (54):

53) le portò cinquecento be' fiorin d'oro (Dec, VIII, 10, 37, p. 1343);

54) de dare per sua tratta, chominciata di ssotto dietro, lb. XJ e s. XIJ dies tre intrante aprile, che nn' èi otto fiorini d'oro belli (Bencivenni, p. 437).

Come in italiano moderno, in italiano antico esistono aggettivi usati in funzione attributiva che, a seconda che siano collocati in posizione prenominale o postnominale, presentano significati diversi, che li fanno rientrare ora nell'ambito dei determinativi ora in quello dei qualificativi: è il caso di semplice, con il duplice significato di 'solo' e 'non complesso, (se riferito a persona) inesperto, ingenuo, modesto, sobrio', e nuovo, che può voler dire 'altro, ulteriore' oppure 'non vecchio'. In italiano moderno, in posizione prenominale semplice e nuovo assumono il primo significato, in posizione postnominale il secondo (una semplice domanda vs una domanda semplice; un nuovo vestito vs un vestito nuovo). In italiano antico, anche in virtù del fatto che un aggettivo con valore restrittivo può trovarsi prima del nome, a differenza che in italiano moderno, possiamo trovarli in posizione prenominale con entrambi i significati (55-56), mentre collocati dopo il sostantivo si comportano solo come qualificativi canonici (57):

55) le portò cinquecento be' fiorin doro, li quali ella ridendo col cuore e piagnendo con gli occhi prese, attenendosene Salabaetto alla sua semplice [= solo verbale, senza ricevuta o dichiarazione scritta] promessione (Dec, VIII, 10, 37, p. 1343);

56) Vedete il re de la semplice [= modesta] vita / seder là solo, Arrigo d'Inghilterra (Purg., VII, 130, p. 119);

57) Egli mi giova molto quando un savio uomo è da una donna semplice [= inesperta, ignorante] menato (Dec, VII, 5, 52, p. 1116).

Una sequenza di aggettivi di tipo pronominale e qualificativo può presentare all'interno del $\mathrm{SN}$ un ordine canonico o ordini marcati. Si può individuare, su indicazione di Giusti (GIA: 607-608), un ordine non marcato sostanzialmente coincidente con quello dellitaliano moderno, eccezion fatta per la posizione dellaggettivo restrittivo, che nella lingua moderna può essere solo postnominale: Art. + altro (o altro aggettivo indefinito) + possessivo + Agg. di quantità o numerale cardinale + Agg. numerale ordinale + Agg. appositivo + Agg. restrittivo $+\mathrm{N}+$ Agg. restrittivo / di relazione. In questa sede ci limiteremo a ricordare alcune possibili sequenze aggettivali. Gli aggettivi appositivi di norma precedono i restrittivi (58). Se un nome è accompagnato da un possessivo e da un qualificativo, la coppia di aggettivi può precedere, come in italiano moderno, o seguire il nome con l'ordine possessivo + qualificativo. In (59) si ha la posizione prenominale; in (60) quella postnominale:

58) Al tempo di Tulio era Salustio, uno grande filosafo maldicente (Fiori, XXI, p. 168);

59) Qui conta come maestro Giordano fu ingannato da un suo falso discepolo (Nov, XI, rubrica, p. 30);

5 Come osserva Cimaglia (EncIt: 667, s.v. Intensificatori). 
60) E in questa maniera son qui, dove io, la buona mercé di Dio e non tua, fratel mio dolce, ti veggio (Dec, II, 5, 23, p. 360).

\subsection{Struttura argomentale dell'aggettivo qualificativo}

In precedenza, si è parlato della topologia dei complementi preposizionali dell'aggettivo e si è detto che al loro posto è possibile trovare proposizioni esplicite ( $E$ chi t'ha fatta certa che quegli che è venuto non sia desso?; Fiammetta, VII, 8, p. 228) o implicite, all'infinito; di norma la preposizione del complemento è la stessa che introduce la frase-complemento all'infinito dipendente dal medesimo aggettivo, come si può osservare a proposito delle preposizioni $a$ in (61)-(62) e di in (63)-(64):

61) Et ben potrebbe il dittatore dicere parole diritte et ornate, ma non varrebbero neente s'elle non fossero aconcie alla materia (Rettorica, p. 151);

62) Le cicogne, quando loro padre e madre per vecchiaia perdono le penne, sicché non sono $a c$ conci a cercare i loro cibi, i figliuoli scaldano le fredde membra (B. da S. Concordio, IV, 8, p. 299);

63) Papirio fue di Roma, omo fortissimo e di grande cuore e desideroso di battaglie (Fiori, XIII, p. 136);

64) desiderosa di vedere qual fosse, [...] vidi infra le frondi un giovanetto palido e timido nello aspetto (Ninfe, XXXII, 53, p. 779).

Un complemento e una proposizione-complemento, come quella limitativa, possono non essere contigui all'aggettivo da cui dipendono: in (65) si ha un altro caso di frase infinitiva non contigua all'aggettivo (l'infinito menar è coordinato ad altri due, sempre retti da usato di: usato [...] di menar [...] e tenervela [...] e [...] mandarla via); in (66) e (67) osserviamo che, come in italiano moderno, il complemento non adiacente all'aggettivo può essere espresso rispettivamente da un sintagma relativo e da un pronome clitico:

65) Dove [= in un casamento] [...] era usato un figliuolo del detto Niccolò, che avea nome Filippo, sì come giovane e senza moglie, di menar talvolta alcuna femina a suo diletto e tenervela un di o due e poscia mandarla via (Dec, IX, 5, 7, p. 1415);

66) di che ella, ogni avversità trapassata dimenticando, divenne lieta (Dec, II, 7, 27, p. 405);

67) il che [= il tema amoroso], se io non erro, per ciò che innamorate credo che siate, molto $v i$ dovrà esser caro (Dec, V, 1, 2, p. 835).

Due complementi coordinati possono entrambi precedere o seguire l'aggettivo che li regge (68) oppure essere collocati in posizione discontinua (69), con la congiunzione coordinante che precede il secondo elemento; qualcosa di simile si era visto già in (28), dove coppie di aggettivi coordinati erano collocate in posizione discontinua rispetto al nome di riferimento: 
68) l'avere veduta Troia d'altissimi palagi e di nobile popolo piena, accesa di greco fuoco e abbattuta tutta (Fiammetta, VIII, 10, p. 242);

69) sopra l'altro canto il palido ulivo, caro a Pallade molto, di rami pieno si vedea e di frondi (Ninfe, XXVI, 33, p. 748).

Molti aggettivi possono anche essere seguiti dalla sequenza, di valore limitativo, $a+$ infinito ( $f a$ cile a dire), laddove in italiano moderno sono più usuali i costrutti con $d a+$ infinito (facile da dire) o a/da + infinito + si passivante (facile a dirsi, facile da dirsi); il soggetto implicito della limitativa è generico e impersonale, mentre l'oggetto implicito dell'infinito «è coreferenziale con il soggetto della predicazione aggettivale» (GIA: 612):

70) Saranno per avventura alcune di voi che diranno che io abbia nello scriver queste novelle troppa licenzia usata, sì come in fare alcuna volta dire alle donne e molto spesso ascoltare cose non assai convenienti né $\boldsymbol{a}$ dire né $\boldsymbol{a}$ ascoltare a oneste donne (Dec, X, Conclusione dell'Autore, 3, p. 1656);

71) Secondamente domandaste: quant'acqua è in mare. Questo mè stato molto forte a vedere (Trecentonovelle, IV, 17, p. 14).

Buono e convenevole ammettono sia $a+$ infinito (costrutto non marcato) che $d a+$ infinito:

72) questo non solamente darà diletto buono a udire, ma sottile amaestramento e a così parlare $\mathrm{e}$ a così intendere l'altrui scritture ( $C v, \mathrm{I}, \mathrm{II}, \mathrm{p} .12)$;

73) la loro carne è molto buona da mangiare (Tesoro Battelli, V, L, p. 162);

74) E Salamon disse: - Perché di' tu, donna, tante parole e cose che non sono convenevoli a dire? (Giamboni Fiore (red. beta), XLVII, p. 51);

75) Filocolo, udite le parole dell'amiraglio, pensa un poco, e prima che risponda, essamina quello che convenevole sia da dire, e che da tacere (Filocolo, IV, 151, 1, p. 539).

Talvolta l'oggetto del verbo all'infinito, generalmente implicito, può essere esplicitato da un pronome clitico, come le nel seguente es.:

76) grandi sono le industrie, e dove non giucassono l'inganni o' tradimenti, care sono a udirle, e ancora a comprenderle (Trecentonovelle, CCXXIV, 10 p. 790).

\subsection{Grado comparativo}

Come in italiano moderno, in italiano antico il grado comparativo di maggioranza, minoranza e uguaglianza è costruito in modo analitico, con l'aggettivo preceduto rispettivamente da più, meno e tanto; il comparativo di uguaglianza può anche essere introdotto dagli avverbi così e sì. Con il comparativo di maggioranza e minoranza, il secondo termine di paragone, se espresso, è introdotto da $d i$ (77) o da che (78); può anche essere frasale, cioè costituito da una proposizione comparativa con verbo di modo finito (79) o indefinito (80); se non espresso, può essere recuperato dal contesto (81) o essere sottinteso (82); può consistere, inoltre, in una qualità espressa da 
un secondo aggettivo e confrontata con quella indicata dal primo aggettivo in riferimento a uno stesso termine (83):

77) tutte le fiere ho trovate piú umili di te (Nov, LXX, 6, p. 120);

78) metti cinquemilia fiorin d'oro de' tuoi, che meno ti deono essere cari che la testa (Dec, II, 9, 22, p. 463);

79) De le compagnie neuna è più graziosa nè più ferma che quando i buoni uomini simiglianti in costumi son giunti di famigliaritade e d'amore (Fiori, XX, p. 154);

80) Neuna cosa è più grave nè più malagevole che tener l'amistade insino a lo stremo die de la vita (Fiori, XIV, p. 141);

81) le cose deono essere denominate da l'ultima nobilitade de la loro forma; sì come l'uomo da la ragione, e non dal senso né d’altro che sia meno nobile (Cv, II, VII, p. 98);

82) E questo è quello per che ciascuno profeta è meno onorato nella sua patria [sott.: che altrove] (Cv, I, IV, p. 19);

83) Ma la mia penna, meno onesta che vaga, s'apparecchia di scrivere quegli ultimi termini d'amore (Fiammetta, I, 24, p. 48).

Il complemento di paragone poteva talora essere espresso da un aggettivo possessivo (Migliorini 2016[1961]: 150):

84) E certo no gli è troppo disinore, / quand'omo è vinto d'un suo [= di lui] migliore (Guido delle Colonne, vv. 22-23, p. 88).

È frequente, inoltre, l'uso del costrutto non meno + Agg. + che / di + secondo termine di paragone (o frase comparativa) o secondo aggettivo, che consente di negare l'idea di minoranza ed esprimere quella di ugual misura ma in modo più approssimativo di quanto permetta il comparativo di uguaglianza:

85) Messer l'abate [...], ancora che vecchio fosse sentì subitamente non meno cocenti gli stimoli della carne che sentiti avesse il suo giovane monaco (Dec, I, 4, 15, pp. 236-237).

Ecco ora alcuni esempi di comparativo di uguaglianza; il secondo termine di paragone o la proposizione comparativa, sempre espressi, sono introdotti da come o quanto:

86) già sotto la luna / non si truova persona / che, per gentil legnaggio / né per altro barnaggio, / tanto degno ne fosse / com'esto re Nanfosse (Tesoretto, II, 134, p. 180);

87) con ciò sia cosa che [...] nulla sia sì intima amistade come da bon padre a bon figliuolo e da bon figliuolo a bon padre [...]; manifesto è che questa donna fue amarissimamente piena di dolore (VN XIII, 2, pp. 116-117). 


\subsection{Grado superlativo}

Come in italiano moderno, il superlativo assoluto si forma con il suffisso -issimo (88) o con l'avverbio di quantità molto (89); a differenza della lingua moderna l'italiano antico conosce anche l'uso combinato dell'avverbio e del suffisso elativo; in (90) troviamo sia molto + Agg. di grado positivo sia molto + Agg. + -issimo:

88) Seneca fue nobilissimo filosafo (Fiori, XXIV, p. 175);

89) Disse il Basso: - Io sono molto contento (Trecentonovelle, XVIII, 7, p. 61);

90) Avea sognato la notte che nel seno li volava un pulcino di molto bianchissimo colore e con molto chiara boce (Fiori, VIII, p. 123).

Oltre a molto, con il superlativo in -issimo è possibile trovare altri intensificatori:

91) tutta la camera del Comune votò, e trassene tra più volte assai bellissime balestra e altri guernimenti da oste (Giovanni Villani, I, VII, p. 392);

92) vidde ch'era savia e si bellissima (Neri Pagliaresi, XI, V, 1, p. 138);

93) chi intendesse bene quella lingua - è troppo bellissima - questo «chere» si è a dire "allégrati» (Quaresimale, LXXII, p. 354; chere in corsivo nel testo).

Sono attestate le forme asprissimo (94) e benevolissimo (95) in luogo dei superlativi di derivazione latina asperrimo e benevolentissimo, in uso oggi; figurano già in italiano antico i superlativi di influsso latino acerrimo, celeberrimo, miserrimo e beneficentissimo, ma è molto comune anche miserissimo (96):

94) la detta cittade è asprissima guardiana di vera giustizia (Val. Max., 69, p. 41);

95) E se tale e tanto è 'l sementatore e 'l seme, caro mio frate, quanta benevolissima reverenzia [...] rendere dovete lui onni tempo? (Guittone Lettere, IX, 9, p. 111);

96) Atto di miserissimo ingegno è sempre usare le cose trovate, e non mai trovarne (B. da $S$. Concordio, I, 2, p. 191).

Tra i superlativi organici dell'italiano antico figura il gallicismo (il) soprano (o sovrano) '(il) più alto; (il) supremo', usato con valore di superlativo assoluto (97) o relativo (98):

97) Cato dice: Soprana virtù è costringere la lingua (Tesoro Gaiter, vol. 3, VII, XIII, p. 253);

98) Nè figlia di Taumante [...] ec ${ }^{6}$. Cioè la folgore, la quale secondo Aristotile si forma di vapore grosso e levato al soprano interstizio dell'aere (Ottimo, II, XXI, p. 390).

Il superlativo relativo si costruisce, in italiano antico come in italiano moderno, con l'articolo determinativo seguito da più o meno e l'aggettivo, oppure con le forme sintetiche (il migliore, il peggiore, il maggiore, il minore); il termine di confronto, se espresso, è introdotto generalmente da

6

In corsivo nel testo. 
di, come in (99), (101) e (104); più raramente da (in)fra / (int)tra, se si vuole marcarne il valore partitivo (100); il termine di paragone può anche consistere in una frase relativa (103); se esso è sottinteso, il superlativo acquista valore universale $(102)^{7}$ :

99) E l'om, se Dio mi vaglia, / crëato fu san' faglia / la più nobile cosa / e degna e prezïosa / di tutte crëature (Tesoretto, VII, 669, p. 199);

100) Et poi regnao Vespasiano $\mathrm{x}$ anni, lo quale infra tutti li boni esso fo lo miliore (Troja Roma, $\mathrm{p}$. 292);

101) E presi li nomi di .lx. le più belle donne de la cittade (VN II, 11, p. 32);

102) Ragione è che 'l capo sia seguitato da' membri: imperò ch'egli è il più nobile membro, è ragione che gli altri membri il seguitino (Avventuale, XXII, p. 318);

103) Intra' morti rimase [...] messer Guiglielmino de' Pazzi di Valdarno e' suoi nipoti, il quale fu $i l$ migliore e 'l più avisato capitano di guerra che fosse in Italia al suo tempo (Giovanni Villani, I, VIII, p. 602);

104) E volendo quivi l'abate albergare, Alessandro in casa d'uno oste [...] il fece smontare, e fecegli la sua camera fare nel meno disagiato luogo della casa (Dec, II, 3, 24, p. 338);

Come mostrato dagli ess. (99) e (101)-(104), la collocazione dellaggettivo di grado superlativo relativo è di norma solo prenominale; l'eventuale termine di confronto, espresso da un complemento o da una frase relativa e indicante l'insieme di riferimento rispetto al quale ha valore la qualità espressa dall'aggettivo, trovandosi in posizione postnominale, è pertanto non contiguo a quest'ultimo.

Sono infine attestate forme di comparativi e di superlativi sintetici usate come aggettivi di grado positivo e quindi precedute da più, per esprimere il comparativo, o il più, per esprimere il superlativo. Questi usi sono oggi considerati per lo più inaccettabili - con qualche rilevante eccezione: le forme registrate in (105), (106) e (108) sono almeno parzialmente accettabili anche in italiano moderno -. Seguono ess. di comparativi organici preceduti da più (105) e il più (106), nonché di superlativi organici preceduti da più - comparativo (107) - e il più - superlativo (109) -:

105) La settima [condizione], della sesta contraria, è se ti chiami più inferiore e più vile dell'́altre non solamente con lingua, ma esiandio coll'intimo affetto del cuore (Simone da Cascina, II, XXI, p. 131).

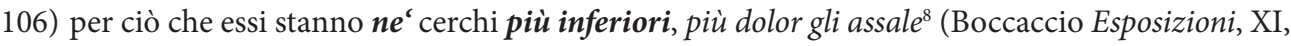
21, p. 542);

107) Avaritia, neuna cosa è più pessima (Jacomi senese, p. 50);

108) né più sommo di lui nelle nostre arti né di maggiore fama alcuno oggi risuona ne’ nostri regni (Ninfe, XVIII, 37, p. 730);

109) Sopra tutte le cose ree l'uomo è la più pessima (B. da S. Concordio, I, 8, p. 351).

7 Anche di un superlativo assoluto in -issimo si può trovare il superlativo relativo, diversamente che in italiano moderno: E come la rosa, il più bellissimo de' fiori, è circondata di spine, cosi Nostra Donna in mezzo de’ peccatori ed infruttiferi, bellissima, delicatissima, e piacevole ad ogni senso apparve (Ottimo, III, XXIII, p. 512). Talvolta il superlativo assoluto è impiegato con valore di superlativo relativo: l'uomo è perfettissimo di tutti li animali (Cv, II, VIII, p. 104).

8 In corsivo nel testo. 


\section{Aggettivi pronominali}

\subsection{Aggettivi possessivi}

Diversamente da altre lingue, come il francese, l'inglese e (in parte) lo spagnolo, in cui la funzione aggettivale e quella pronominale sono affidate a due serie diverse di possessivi, l'italiano conosce una sola serie di elementi, che possono svolgere l'una o l'altra funzione a seconda che il possessivo accompagni o meno un nome (Serianni 2010[1989]: 267).

In italiano antico l'aggettivo possessivo può precedere (110) o seguire il nome cui si riferisce (111). Come in italiano moderno, anche nei testi antichi la posizione più frequente e non marcata è quella preverbale (110); quella postverbale (111), tuttavia, non presuppone necessariamente la focalizzazione dell'aggettivo, cosa invece molto comune in italiano moderno (Serianni 2010[1989]: 269-271). In (112), che esibisce la compresenza di entrambe le posizioni, la posposizione di mio enfatizza retoricamente la minaccia veicolata dallenunciato, sebbene non si possa affermare con certezza che l'elemento sia focalizzato9:

110) E ponete vostre ancore en fondo de xvij passi entro en xxx passi (Compasso, p. 81);

111) Cessino questo gl'iddii, che un re si possa dire che colpevole nella morte d'una semplice giovinetta sia, o che le mani vostre di sì vile sangue siano contaminate (Filocolo, III, 40, 3, p. 317);

112 ) io ti farò conciare in maniera, che tu con tuo danno ti ricorderai, sempre che tu ci viverai, del nome mio (Dec, VI, 4, p. 13).

Quando si trova in posizione prenominale, l'aggettivo possessivo tende a precedere eventuali altri aggettivi o modificatori del nome (113-114); in posizione postnominale, esso può essere accompagnato sia da un aggettivo prenominale (115): si può notare la coesistenza di due figurazioni diverse del SN), sia da un aggettivo postnominale (116) o un SP che lo segue (117):

113) Trovamo ciascheduno planeta èssare portato enverso oriente da uno suo grande cerchio, lo quale è chiamato deferente (Restoro, I, 12, 3, p. 31);

114) E questo mio primo amico e io ne sapemo bene di quelli che così rimano stoltamente ( $V N$, XVI, 10, pp. 155-156);

115) Al karissimo e dilectissimo figliuolo mio di merito e non di peccato, e senza aspectamento de heredità sempiterna, paterna benedictione. Sappiate che io a te, benedetto mio figliuolo, t'avrei sovente tutto tuo conforto scritto (Sommetta, p. 206);

116) Et io prego lo sposo vostro diletto che con plenitudine ad voi si dia e in voi si trasformi (Colombini, XL, p. 133);

117) Dunque la doctrina tua del'amore et dela dilectione di Dio prenda cominciamento (Albertano volg., I, 3, p. 55).

9 In questa sede si considera il focus, in un'accezione ristretta, come categoria discreta, non presente in tutti gli enunciati e distinta da altri tipi di salienza, come secondo la visione di Hammarström (2012). Sulle frasi marcate in italiano antico cfr. anche il recente volume di Nicolosi (2018). 
In italiano antico, l'aggettivo possessivo può avere forma ridotta (GIA: 359-360, 1404-1406). Il troncamento, come osserva Penello (GIA: 1404), si realizza in due serie di forme diverse: miè, tuò, suò, usata per il plurale, «la cui riduzione dipende da motivazione fonologica»; mi' (anche nella variante $m e$ ', senza innalzamento di e pretonica), $t u$ ', $s u$ ', «senza motivazione fonologica». Se la prima serie ha valore esclusivamente plurale, la seconda, comune al fiorentino moderno (Rohlfs 1966-69: II, 121) e ad altri dialetti dell'Italia centrale - per es. il romanesco, ma solo con nomi di parentela che prevedono la presenza del possessivo anteposto senza articolo: mi' padre, tu' madre, su' fratello -, in italiano antico può avere valore sia singolare, sia plurale:

118) Laudato sie, $m i$ ' Signore, cum tucte le tue creature ( $L C$, v. 5, p. 33);

119) Una mi prese e tiemmi con sua artigli, / per chella vide sùbite mi' voglie (Tedaldi, X, v. 6, p. 726);

120) E poscia ch'io n'avessi tanti tolti, / ch'a me 'l $\boldsymbol{t} \boldsymbol{u}$ ' pianto fosse discoperto, / morte vorrei dalle tua man, per certo (Boccaccio Rime, II, 15, vv. 5-7, p. 168);

121) Le paion fatte al torno / oimè le $\boldsymbol{t} \boldsymbol{u}^{\prime}$ belleççe (Mortara, vv. 113-4, p. 95).

Le prime tre persone, come mostrano gli esempi, presentano l'apocope della vocale finale. Sebbene tale caratteristica possa riguardare anche il pronome (122), essa è più frequente quando il possessivo ha funzione aggettivale (118-121):

122) che se per $\boldsymbol{t} \boldsymbol{u}^{\prime}$ conforto / il $\boldsymbol{s} \boldsymbol{u}^{\prime}$ disperde a torto / e torna in basso stato, / tu ne sarai biasimato (Tesoretto, vv. 1699-1702, p. 235).

Laggettivo possessivo, infine, può apparire senza articolo, anche in contesti in cui l'italiano moderno lo prevederebbe:

123) dalla viva fonte del suo ingegno fece suo libro di rettorica (Rettorica, p. 6).

\subsection{Aggettivi indefiniti}

I cosiddetti aggettivi indefiniti rientrano nella classe dei quantificatori. Tre sono le differenze più rilevanti tra l'italiano moderno e l'italiano antico: 1) la presenza o l'assenza dell'articolo nella configurazione del SN; si osservi, in (124), la compresenza di due forme concorrenti nello stesso testo, per di più a breve distanza; 2) la posposizione, in italiano antico, di aggettivi che nella lingua doggi, strutture marcate a parte, precedono sistematicamente il sostantivo di riferimento (125); 3 ) il plurale, in italiano antico, di aggettivi che l'italiano moderno conosce solo nella forma singolare (126):

124) 'Mazzeo genitore e l'amato suo figliuolo P. salute e in tutte buone cose la parterna benedictione', vel 'salutem e di bene in meglio procedere e bastamente acrescere', vel 'salutem e la benedictione perfecta, la quale diede Ysaàc e Iacòb suo figliuolo di crescere e multiplicare', vel 'salutem con adornamenti di graziosi costumi, sicché possi a Dio e a li huomini piacere', vel 'salutem e perfectione di compiuta scienzia per venire in tutte le buone cose e habundare' (Sommetta, p. 203); 
125) Onde disse Salustio: sempre nelle cittadi ne le quali nonn' à ricchezze alcune, è avuto invidia de' buoni da coloro ke non sono ricchi (A. Da Grosseto Albertano, IV, 18, p. 336);

126) E altri giovani cardinali fatti per papa Clemento erano stati, e in questi dì erano in tanta disoluta vita, che niuni giovani disoluti tiranni li avanzavano (Matteo Villani, IV, 86, p. 599).

\subsection{Aggettivi dimostrativi}

Litaliano antico ha un ventaglio di dimostrativi più ampio di quello dell'italiano moderno, sia come inventario di forme sia come varietà d'uso. Da segnalare, innanzi tutto, è la presenza di esto (127), con valore esclusivamente aggettivale (GIA: 350), che continua il lat. ǏstŭM. Codesto (128), anche nella più frequente - nella variante cotesto (130), con dentale sorda, oggi vitale solo in area toscana, affianca questo (129) e quello (130), a completare il quadro dei deittici (Rohlfs 1966-1969: II, 202-204):

127) Lo mar potresti arompere, / a venti asemerare, / l'abere d'esto secolo tutto quanto asembrare (Cielo d'Alcamo, vv. 7-8, p. 178);

128) ti scongiuro per Dio, che se tu se' omo o creatura ragionevole chabiti in codesta spelonca, che tu mi risponda e spianami la verità (Leggenda Aurea, vol. II, XCI, p. 799);

129) E di questo uno exemplo si puote intendere tutti i somiglianti (Rettorica, p. 163);

130) mi par pur vederti morderle con cotesti tuoi denti fatti a bischeri quella sua bocca vermigiuzza e quelle sue gote che paion due rose (Dec, IX, 5, p. 609).

Come in italiano moderno, l'aggettivo dimostrativo si colloca al principio del SN di cui fa parte, precedendo altri eventuali aggettivi, come i possessivi (130); in (129) si osserva la presenza del numerale uno, con cui il dimostrativo dell'italiano moderno non è più compatibile (GIA: 350$)$. Fa eccezione il quantificatore universale tutto, che precede anche i dimostrativi:

131) Trovate nella causa tutte queste cose (Rettorica, p. 139).

\subsection{Aggettivi relativi}

Anche i relativi, classificati tradizionalmente come pronomi, possono assumere, occasionalmente, ruolo aggettivale, tanto in italiano antico (Bianco 2009, SIA: 257-258) quanto in italiano moderno (Bianco 2012):

132) E poi fo mandato contra Mitridate êlle parti de Costantinopoli, el quale Mitridate avea bene .XL anni guerra colli Romani avuta, e de nocte in tal guisa l' asalse a li padillioni che, senza danno de li suoi cavalieri, de quelli de Mitridate ucise .XX. milia (AC, V, 13-18, p. 79);

133) La terzogenita Elvira in questo modo non aveva mai potuto conoscere suo padre: grande motivo per lei di tormento e frustrazione. Il quale padre all'inizio scriveva, ogni tre-quattro-cinque anni: delle lettere brevi, inframmezzate da parole straniere, non in inglese ma in spagnolo (Campailla, pp. 78-79). 
Come mostrano gli esempi, l'aggettivo relativo assume la forma di un relativo analitico seguito da un SN ad esso accordato morfologicamente; quest'ultimo stabilisce con il cotesto precedente un nesso di varia natura. Laggettivo relativo introduce sempre una frase con valore appositivo (GIA: 488); spesso, come in (134), ricorre nella cosiddetta coniunctio relativa, al punto da essere quasi identificato con essa:

134) Occise Bella frate sio e fu sconfitto da Franceschi, Borgognoni e Sanzonesi e Italiani. Nella quale sconfitta fu muorto lo re de Borgogna (AC, 18, 894-898, p. 173).

Piuttosto ampio è il ventaglio dei possibili rapporti fra il SN di cui fa parte il relativo e il proprio antecedente lessicale o testuale:

135) La terza scienzia, cioè teorica, si è per dimostrare le nature di tutte cose che sono, le quali nature sono tre (Rettorica, p. 44);

136) E stando noi $\boldsymbol{e}$-lla cità d'Arezzo, e.lla quale noi fommo nati, e.lla quale noi facemmo questo libro, la quale cità è posta enverso la fine del quinto clima (Restoro, I, 15, 3, p. 22);

137) Ancora si muove tutto questo cielo e rivolgesi collo epiciclo da oriente in occidente, ogni die naturale una fiata: lo qual movimento, se esso è da intelletto alcuno, o se esso è dalla rapina del Primo Mobile, Dio lo sa (Cv, II, 5, pp. 91-92);

138) Nel detto anno e mese di luglio ne la città di Pisa era ordinata cospirazione, ond'era capo messer Gherardo del Pellaio de' Lanfranchi, per cagione che a llui e alla sua setta parea che quegli che reggeano la terra fossono contra parte imperiale, e tenessono troppo colla Chiesa e co' Fiorentini, overo per invidia de la signoria. La quale congiura scoperta, il detto messer Gherardo e più suoi seguaci si partirono di Pisa, e furono condannati per rubelli, e IIII popolani che ne furono presi come traditori furono impiccati (Compagni, XI, CLXI, p. 723);

139) La quale cosa avere non si puote sanza prudenza e sanza giustizia; le quali virtudi anzi a questa etade avere perfette per via naturale è impossibile ( $C v, I V, 27$, p. 438-439);

140) Nel detto anno MCCCIIII, a dì XXV del mese di luglio, essendo la città di Firenze in tante aversitadi e fortune, gli Aretini cogli Ubertini e' Pazzi di Valdarno vennero con tutto loro podere di gente d'arme a cavallo e a piede al castello di Laterino, il quale teneano i Fiorentini, e aveano tenuto lungo tempo per forza, e quello col'aiuto de' terrazzani fu loro dato; e la rocca la quale aveano fatta fare i Fiorentini, l'avea in guardia messere Gualterotto de' Bardi, perch'era venuto a Firenze per le novitadi che v'erano state, convenne s'arrendesse pochi dì appresso, però chera rimasa mal fornita, e per le novità di Firenze non aspettavano soccorso. E alcuno disse che gli Ubertini suoi parenti il ne tradiro e ingannaro, e chi disse che lo 'nganno fu fatto al Comune. De la quale perdita del castello spiacque molto a' Fiorentini, però chera molto forte, e in una contrada che tenea molto a freno gli Aretini (Compagni, IX, LXXIII, 1-18, pp. 140-141);

141) ed essendo già terza ed essi alla città pervenuti, avvisando d’essere al migliore albergo inviati, con messer Torello alle sue case pervennero, dove già ben cinquanta de' maggiori cittadini eran venuti per ricevere i gentili uomini, a' quali subitamente furon dintorno a' freni e alle staffe. La qual cosa il Saladino e'compagni veggendo, troppo s'avvisaron ciò che era (Dec, X, 9, 22-23, p. 1211); 
142) E del suo [= del marchese di Monferrato] valore ragionandosi nella corte del re Filippo il bornio, il quale a quello medesimo passaggio andar di Francia s'aparecchiava, fu per un cavalier detto non essere sotto le stelle una simile coppia a quella del marchese e della sua donna; però che, quanto tra' cavalieri era d'ogni virtù il marchese famoso, tanto la donna tra tutte l'altre donne del mondo era bellissima e valorosa. Le quali parole per sì fatta maniera nell'animo del re di Francia entrarono, che, senza mai averla veduta, di subito ferventemente la cominciò ad amare (Dec, I, 5, 6-7, pp. 90-91).

In (135) e (136) si osserva la semplice ricorrenza di un antecedente lessicale; nel caso in cui quest'ultimo sia costituito da un sintagma polirematico, accade frequentemente che il SN di ripresa recuperi solo un elemento di tale sintagma: non di rado l'apposizione (cità), come in (136); la ricorrenza può essere anche parziale (137), talora con salto di categoria grammaticale $(\mathrm{V} \rightarrow \mathrm{N}$ deverbale), in particolare quando deve essere ripreso un verbo coniugato (si muove $\rightarrow$ lo qual movimento). La ripresa di un antecedente lessicale può avvenire anche a mezzo di un sinonimo (138) o di un iperonimo: (139) mostra addirittura la ripresa di due coiponimi (prudenza, giustizia) per mezzo di un unico SN (virtudi). L'antecedente, infine, può essere anche costituito da un'intera frase o da un blocco di testo più ampio, ripresi per mezzo di un incapsulatore (141-142). Spicca, per frequenza, il sostantivo cosa, nome generale per eccellenza, che nella formula cristallizzata (Prep. +) la qual cosa trova largo uso ancora oggi.

Rispetto all'italiano moderno, tre sono le caratteristiche principali dell'italiano antico quanto all'uso dell'aggettivo relativo: a) una generale maggior frequenza, pur con differenze significative fra i generi testuali e fra i singoli testi (Bianco 2009: 45-49; 2012: 310); b) una più ampia varietà di tipi di ripresa: le ricerche compiute mostrano come in italiano contemporaneo sia pressoché preclusa la possibilità della ricorrenza parziale (Bianco 2012: 310-311) e la ripresa di segmenti testuali avvenga, quasi sempre, per mezzo di formule fisse (Bianco 2012: 312); 4) la possibilità di riprendere tanto antecedenti facili, per es. SN immediatamente precedenti l'aggettivo relativo, quanto antecedenti "difficili", vuoi per la distanza, vuoi per l'architettura sintattica della frase, vuoi per la loro forma. Nei testi antichi, l'aggettivo relativo (che costituisce, assieme al sostantivo di ripresa, un doppio legame con il cotesto precedente: grammaticale e semantico) rientra fra le risorse atte a realizzare un ideale di prosa "legata"; in italiano moderno, invece, è il contesto, linguistico o extralinguistico (Bianco 2012:314), a determinare la possibilità di ricorrere all'aggettivo relativo quale strategia di ripresa più forte di altre, più canoniche.

Un'ultima differenza, infine, riguarda la struttura: la forma canonica dell'aggettivo relativo, come si è detto, è Art. + quale/ $i+\mathrm{SN}$; in italiano antico, tuttavia, può capitare, seppur raramente, che venga a mancare l'articolo determinativo, cosa impossibile in italiano moderno:

143) Ma dicie lo chonto che alquanto Choragino, da parte de donna, era parente de Ulisste e perciò, al passare del mare, Choragino s'achonpangnò chon Ulisste e senpre Choragino era ad una chorte chon Ulisste e ssuo fratello e Forandano; quale Forandano fo bailo de Choragino (Corciano, 1, 28-29-2, 1-3, p. 30). 


\section{Altri modificatori del nome}

\subsection{Sintagmi preposizionali}

Come osserva Frenguelli (2020: 606), «il SP [...] è in grado di modificare tutte le categorie lessicali, ad eccezione dei clitici». In particolare, i SP possono essere usati in sostituzione degli aggettivi come modificatori del nome:

144) Imbestiate schegge fo quello istrumento ligneo (Lana, p. 1488);

145) Quand’ànno a fare l'uno coll'altro, fanno tacche di legno (Milione, 119, p. 189).

Il SP attributivo può svolgere vari ruoli semantici: (145) esibisce un complemento di materia, mentre gli ess. seguenti presentano, rispettivamente, complementi di argomento (146) e di qualità $(147)^{10}$ :

146) Incominciasi il libro de' Vizî e delle Virtudi e delle loro battaglie e ammonimenti (Giamboni Libro, I, rubrica, p. 3).

147) Fu miserabile e cordoglioso riguardo e aspetto di gran crudeltà vedere li vecchi pieni d’anni, le donne, le fanciulle lagrimose co- sospiri e guai, e lli piccioli fanciulli conn istrida lasciare loro case (Giovanni Villani, XI, XVI, p. 608).

Possono altresì trovarsi svolgere funzione predicativa, tanto retti da essere (148) quanto da altri verbi copulativi (149):

148) Ma l'altre anime sono con peccato (Prediche, 4, p. 53);

149) E parevagli che questi tenesse nella sinistra uno arco bellissimo e forte, e nella destra due saette, l'una d'oro, e quella era argutissima e pungente, l'altra gli parea di piombo, sanza alcuna punta (Filocolo, III, 19, 2, pp. 275-276).

\subsection{Frasi relative}

Come in italiano moderno, la FR (SIA: 196-269) con antecedente può essere usata per modificare un nome, come relativa restrittiva; come si è detto in apertura, invece, non è possibile usarla come elemento predicativo di un predicato nominale:

150) Avea questo una soa moglie moito iovine e bella, la quale, quanno iva a Santo Pietro, iva accompagnata da iovini armati (AR, XVIII, 1015, p. 177);

151) Dice Tulio che quella questione iudiciale del genere èe appellata assoluta la quale in sé medesima è disciolta e dilibera (Rettorica, p. 97);

10 Trattazioni più dettagliate delle funzioni svolte dal SP in italiano antico sono offerte da Andreose (GIA: 617-714) e da Frenguelli (2020: 606-630). 
152) E stando schierati i cavalieri, e fu presso che finita la guerra; tanto scandalo nacque tra quelle genti. Il quale se fusse ito innanzi, i grandi e il popolo, a cui piacea la pace, amici del Cardinale, n’arebbono avuto il migliore, secondo che le volontà si dimostravano (Compagni, III, VI, 293, p. 94).

Non di rado (151), il SN costituito dall'antecedente e dalla relativa è discontinuo: ciò accade anche in italiano moderno, dove tuttavia la recuperabilità dell'antecedente è limitata (GIA: 504). (152) esibisce una frase sintatticamente indipendente o semi-indipendente, nell'ambito di quel costrutto chiamato nesso relativo, coniunctio relativa o relatif de liaison (Bianco 2009: 31); spesso, in questo caso, il relativo è usato come aggettivo.

\section{Conclusioni}

Losservazione del comportamento - forme, usi, funzioni - del SA in italiano antico costituisce un punto di vista oltremodo interessante sulla sintassi delle fasi più antiche della nostra lingua. Ciò è dovuto, in buona parte, alla natura particolarmente "flessibile" di tale elemento, che può far parte tanto del SN quanto, con valore predicativo, del sintagma verbale.

Muovendo da quella che può essere considerata la forma canonica del SA, vale a dire l'aggettivo qualificativo-relazionale, cui è dedicata la parte centrale della trattazione, abbiamo cercato di considerare funzioni sintattiche analoghe svolte da altri costituenti della frase, dagli aggettivi pronominali fino alle proposizioni relative. Lanalisi di questo vario insieme di strutture, nel confronto fra italiano antico e italiano moderno, suggerisce almeno un tratto comune ai costrutti considerati: la maggior flessibilità del SA antico, parte di una lingua ancora libera dal giogo della codificazione normativa, rispetto al SA moderno. Rari sono i casi in cui possibilità offerte al parlante/scrivente moderno sono sconosciute o interdette a quello dei primi secoli; il rapporto fra le possibili opzioni offerte dal codice si presenta, piuttosto, in termini di maggiore o minore frequenza, eventualmente influenzata da parametri esterni (per es. la provenienza geografica dello scrivente) o interni (funzione svolta dal costituente nel contesto frasale o testuale).

Si tratta di una tendenza, coerente con le conoscenze della lingua antica, confermata a vari livelli: morfologico (si pensi, per es., alle forme del comparativo e del superlativo), topologico, combinatorio (aggettivo + molto), funzionale (SP con valore predicativo), testuale (i vari tipi di ripresa dell'aggettivo relativo). 


\section{Riferimenti bibliografici}

\section{Bibliografia primaria}

AC = Conti di antichi cavalieri, a cura di A. Del Monte. (1972). Milano: Cisalpino-Goliardica.

A. Da Grosseto Albertano = Andrea da Grosseto, Dei Trattati morali di Albertano da Brescia, volgarizzamento inedito fatto nel 1268 da Andrea da Grosseto, a cura di F. Selmi. (1873). Bologna: Commissione per i testi di lingua, Romagnoli.

Albertano volg. = Il Trattato della Dilezione d'Albertano da Brescia nel codice II IV 111 della Biblioteca Nazionale Centrale di Firenze, a cura di P. Larson \& G. Frosini. (2012). Firenze: Accademia della Crusca.

$A R=$ Anonimo romano, Cronica, a cura di G. Porta. (1979). Milano: Adelphi.

Avventuale $=$ Giordano da Pisa, Avventuale fiorentino 1304, a cura di S. Serventi. (2006). Bologna: il Mulino.

B. da S. Concordio = Bartolomeo da San Concordio, Ammaestramenti degli antichi latini e toscani, a cura di V. Nannucci. (1840). Firenze: Ricordi.

Bartolomeo Chirurgia = Maestro Bartolomeo, Volgarizzamento della Chirurgia di Ruggero di Giovanni Frugardo. (2010). In Elena Artale-Miriam Panichella, Un volgarizzamento toscano della Chirurgia di Ruggero Frugardo. Bollettino dell'Opera del vocabolario italiano, 15, 227-298 [testo 234-298].

Bencivenni $=$ Libricciolo di crediti di Bene Bencivenni (Secondo). In Nuovi testi fiorentini del Dugento, a cura di A. Castellani. (1952). Firenze: Sansoni. 363-458.

Boccaccio Esposizioni = Giovanni Boccaccio, Esposizioni sopra la Comedia di Dante, a cura di G. Padoan. (1965). In Boccaccio Opere, VI.

Boccaccio Opere $=$ Tutte le opere di Giovanni Boccaccio, a cura di V. Branca, 10 voll. (1964-1998). Milano: Mondadori.

Boccaccio Rime = Giovanni Boccaccio, Rime. In Id., Rime. Caccia di Diana, a cura di V. Branca. (1958). Padova: Liviana. 3-240.

Bondie Dietaiuti = Bondie Dietaiuti, Rime. In Poeti fiorentini del Duecento, a cura di F. Catenazzi. (1977). Brescia: Morcelliana. 111-152.

Campailla = Sergio Campailla, Romanzo americano. (1994). Milano: Rusconi.

Capitolo = Jacopo Alighieri, Capitolo sopra la Commedia. (1888). In Francesco Roediger, Dichiarazione poetica dell'Inferno dantesco di frate Guido da Pisa. Il Propugnatore, nuova serie, I, 1, 62-92; 326-395 [testo 363-370].

Ciampolo = L'Eneide di Virgilio volgarizzata nel buon secolo della lingua da Ciampolo di Meo degli Ugurgeri senese, a cura di A. Gotti. (1858). Firenze: Le Monnier.

Cielo d'Alcamo $=$ Cielo d'Alcamo, Rosa fresca aulentissima. In PD, I, 177-185.

Colombini = Le lettere del Beato Gio. Colombini da Siena, a cura di A. Bartoli. (1856). Lucca: Balatresi.

Compagni $=$ Dino Compagni, Cronica, a cura di D. Cappi. (2000). Roma: Istituto storico italiano per il Medio Evo.

Compasso = Lo Compasso de navegare. Edizione del codice Hamilton 396 con commento linguistico e glossario, a cura di A. Debanne. (2011). Bruxelles: Peter Lang.

Corciano = Il conto di Corciano e di Perugia, a cura di F. Mancini. (1979). Firenze: La nuova Italia.

Crescenzi $=$ Anonimo, Volgarizzamento del Trattato d'agricoltura di Pietro de' Crescenzi, ridotto a migliore lezione da Bartolomeo Sorio, 3 voll. (1851-1852). Verona: Vicentini e Franchini, [libri I-III; V-XII].

$C v=$ Dante Alighieri, Convivio, a cura di F. Brambilla Ageno. (1995). Firenze: Le Lettere. 
Dec $=$ Giovanni Boccaccio, Decameron, a cura di V. Branca. (1992). Torino: Einaudi.

Fiammetta = Giovanni Boccaccio, Elegia di Madonna Fiammetta, a cura di F. Ageno. (1954). Paris: Tallone.

Filocolo $=$ Giovanni Boccaccio, Filocolo , a cura di A. E. Quaglio. (1967). In Boccaccio Opere, I, 45-675.

Fiori = Fiori e vita di filosafi e d'altri savi e d'imperadori, a cura di A. D’Agostino. (1979). Firenze: La Nuova Italia.

Genesi = Giordano da Pisa, Prediche sul secondo capitolo del Genesi, a cura di S. Grattarola. (1999). Roma: Istituto storico domenicano.

Giamboni Libro = Bono Giamboni, Il libro de' Vizî e delle Virtudi e delle loro battaglie e ammonimenti. (1968). In Id., Il libro de' Vizî e il Trattato di virtù e di vizî, a cura di C. Segre. Torino: Einaudi, 3-120.

Giovanni Villani = Giovanni Villani, Nuova Cronica, a cura di G. Porta, 3 voll. (1990-1991). Parma: Fondazione Pietro Bembo/Ugo Guanda.

Guido da Pisa = Guido da Pisa, Fiore d'Italia, a cura di L. Muzzi. (1824). Bologna: Romano Turchi.

Guido delle Colonne $=$ Guido delle Colonne, Amor, che lungiamente mài menato, a cura di C. Calenda. (2008). In Poeti della corte di Federico II, a cura di C. Di Girolamo. Milano: Mondadori, 53-108 [testo 87-90].

Guittone Lettere = Guittone d'Arezzo, Lettere, a cura di C. Margueron. (1990). Bologna: Commissione per $\mathrm{i}$ testi di lingua.

Jacomi senese $=$ M. Salem Elsheikh, Di uno o più Stricca senesi. Studi danteschi. (1971). 48, 45-66 [testo 48-51].

Lana = Iacomo della Lana, Commento alla 'Commedia', a cura di M. Volpi. (2009). Roma: Salerno.

$L C=$ Francesco d'Assisi, Laudes creaturarum. In PD, I, 29-34.

Leggenda Aurea = Beato Iacopo da Varagine, Leggenda Aurea. Volgarizzamento toscano del Trecento, a cura di A. Levasti, 3 voll. (1924-1926). Firenze: Libreria editrice fiorentina.

Matteo Villani = Villani Matteo, Cronica, con la continuazione di F. Villani, a cura di G. Porta, 3 voll. (1995). Parma: Fondazione Pietro Bembo/Ugo Guanda.

Mazzeo = Mazzeo di ser Bellebuoni, Storia della distruzione di Troia . In Testi inediti di storia trojana, a cura di E. Gorra. (1887). Torino: Loescher, 443-457; 518-523.

Milione = Marco Polo, Il Milione. Versione toscana del Trecento, a cura di V. Bertolucci Pizzorusso. (1975). Milano: Adelphi.

Mortara $=$ P. Allegretti, Un laudario ritrovato: il codice Mortara (Cologny, Bibliotheca Bodmeriana Ms. 94). Studi di filologia italiana. (2002). 60, 35-102.

Neri Pagliaresi = Neri Pagliaresi, Leggenda di santo Giosafà. (1965). In Cantari religiosi senesi del Trecento, a cura di G. Varanini. Bari: Laterza, 7-189.

Ninfe = Giovanni Boccaccio, Comedia delle ninfe fiorentine (Ameto), a cura di A. E. Quaglio (1964). In Boccaccio Opere, II, 678-835.

Nov = Il Novellino, a cura di A. Conte. (2001). Roma: Salerno.

Orosio $=$ Bono Giamboni, Delle storie contra i Pagani di Paolo Orosio, a cura di F. Tassi. (1849). Firenze: Baracchi.

Ottimo = L'Ottimo commento della Divina Commedia. Testo inedito d'un contemporaneo di Dante, a cura di A. Torri, 3 voll. (1827-1829). Pisa: Capurro.

PD = Poeti del Duecento, a cura di G. Contini, 2 voll. (1960). Milano-Napoli: Ricciardi.

Purg. = Dante Alighieri, La Commedia secondo lantica vulgata, a cura di G. Petrocchi, III, Purgatorio. (1994). Firenze: Le Lettere. 
$P G=$ Poeti giocosi del tempo di Dante, a cura di M. Marti. (1956). Milano: Rizzoli.

Prediche = Giordano da Pisa, Prediche inedite (dal ms. Laurenziano, Acquisti e Doni 290), a cura di C. Iannella. (1997). Pisa: ETS.

Quaresimale = Giordano da Pisa, Quaresimale fiorentino 1305-1306, a cura di C. Delcorno. (1974). Firenze: Sansoni.

Restoro = Restoro D’Arezzo, La composizione del mondo colle sue cascioni, a cura di A. Morino. (1997). Parma: Fondazione Pietro Bembo/Ugo Guanda.

Rettorica $=$ Brunetto Latini, Rettorica , cura di F. Maggini. (1968[1915]). Firenze: Le Monnier.

RVF = Francesco Petrarca, Canzoniere, a cura di M. Santagata. (2004). Milano: Mondadori.

Simone da Cascina = Simone da Cascina, Colloquio spirituale, a cura di F. Dalla Riva. (1982). Firenze: Olschki.

Simintendi Volg. Metamorfosi = Arrigo Simintendi, I primi cinque libri. Cinque altri libri. Gli ultimi Cinque libri delle Metamorfosi d'Ovidio volgarizzate da Ser Arrigo Simintendi da Prato, a cura di C. Segre. In La prosa del Duecento, a cura di C. Segre \& M. Marti. (1959). Milano-Napoli: Ricciardi, 511-519.

Sommetta = I. Hijmans-Tromp, La Sommetta falsamente attribuita a Brunetto Latini. Cultura Neolatina. (1999). 59, 3-4, 177-243.

Troja Roma = E. Monaci, Storie de Troja e de Roma. (1920). Roma: Società romana di storia patria.

Tedaldi $=$ Pieraccio Tedaldi, Rime. In $P G, 717-57$.

Tesoretto $=$ Brunetto Latini, Il Tesoretto. In PD, II, 175-277.

Tesoro Battelli = Anonimo, Tesoro di Brunetto Latini volgarizzato [Libri III, IV e V]. In B. Latini, I libri naturali del „Tesoro" emendati colla scorta de' codici, commentati e illustrati da Guido Battelli. (1917). Firenze: Successori Le Monnier, 3-51; 55-72; 75-192.

Tesoro Gaiter = Anonimo, Il Tesoro di Brunetto Latini volgarizzato da Bono Giamboni, a cura di L. Gaiter, 4 voll. (1878-1883). Bologna: Romagnoli.

$T R=$ La Tavola Ritonda, a cura di M.-J. Heijkant. (1997). Milano-Trento: Luni.

Trattati Albertano $=$ F. Faleri, Il volgarizzamento dei trattati morali di Albertano da Brescia secondo il 'codice Bargiacchi' (BNCF II.III.272). Bollettino dell'Opera del vocabolario italiano. (2009). 14, 187-368 [testo 199-368].

Trecentonovelle $=$ Franco Sacchetti, Il Trecentonovelle, a cura di V. Marucci. (1996). Roma: Salerno.

Val. Max. = Il volgarizzamento B del secondo libro [di Valerio Massimo] secondo Vat e FL/3. In Un Volgarizzamento inedito di Valerio Massimo, a cura di V. Lippi Bigazzi. (1996). Firenze: Accademia della Crusca, 1-70.

Vegezio $=$ Bono Giamboni, Dell'arte della guerra di Vegezio Flavio volgarizzata libri IV, a cura di F. Fontani. (1815). Firenze: Marenigh.

VN = Dante Alighieri, Vita Nova, a cura di G. Gorni. (1996). Torino: Einaudi.

\section{Bibliografia secondaria}

Bianco, F. (2009). Laggettivo relativo in italiano antico. Bollettino dell'Atlante Lessicale degli Antichi Volgari Italiani, 2, 31-54.

. (2012). Laggettivo relativo fra italiano antico e moderno. In P. Bianchi et al. (a cura di), La variazione nell'italiano e nella sua storia. Varietà e varianti linguistiche e testuali. Atti dell'XI Congresso della Società Internazionale di Linguistica e Filologia Italiana (Napoli, 5-7 ottobre 2010) (pp. 309-318). 2 voll. Firenze: Cesati. 
Bortolotto, L. (2016). The Syntax of Relational Adjectives in Romance: a Cartographic Approach. Tesi di dottorato. Venezia: Università Ca' Foscari.

Brinker, J. H. (1974). L'aggettivo di relazione nell'italiano moderno. In M. Medici, \& A. Sangregorio (a cura di), Fenomeni morfologici e sintattici nellitaliano contemporaneo (pp. 5-19). Roma: Bulzoni.

Coletti, V. (2018). L'italiano scomparso. Grammatica della lingua che non c’e più. Bologna: il Mulino.

Dardano, M. (1969). Lingua e tecnica narrativa nel Duecento. Roma: Bulzoni. . (2009). Il progetto ArSIL. In A. Ferrari et al. (a cura di), Sintassi storica e sincronica dellitaliano. Subordinazione, coordinazione, giustapposizione. Atti del X Congresso della Società internazionale di linguistica e filologia italiana (Basilea, 30 giugno-3 luglio 2008). (pp. 327-338). 3 voll. Firenze: Cesati.

Dardano, M.; \& Trifone, P. (1997). la Nuova grammatica della lingua italiana. Bologna: Zanichelli.

EncIt $=$ R. Simone (a cura di). Enciclopedia dell'italiano, 2 voll. (2010-2011). Roma: Istituto della Enciclopedia Italiana.

Frenguelli, G. (2020). Il sintagma preposizionale. In M. Dardano (a cura di), Sintassi dellitaliano antico. La prosa del Duecento e del Trecento. La frase semplice (pp. 593-637). Roma: Carocci.

GGIC = Renzi, L.; Salvi, G.; \& Cardinaletti, A. (a cura di) (2001). Grande grammatica italiana di consultazione. 3 voll. Bologna: il Mulino.

GIA = Salvi, G.; \& Renzi, L. (a cura di). (2010). Grammatica dell'italiano antico. 2 voll. Bologna: il Mulino.

Hammarström, G. (2012). Word order and focus in Old and Modern French. Some viewpoints. In B. Wehr, \& F. Nicolosi (a cura di), Pragmatique historique et syntaxe/Historische Pragmatik und Syntax (pp. 93-111). Frankfurt am Main: Peter Lang.

Migliorini, B. (2016 [1961]). Storia della lingua italiana. Milano: Bompiani.

Mortara Garavelli, B. (1988). Manuale di retorica. Milano: Bompiani.

Nicolosi, F. (2018). Topic- und Focus-Markierung im Altitalienischen. Berlin-Boston: de Gruyter.

Rohlfs G. (1966-1969). Grammatica storica della lingua italiana e dei suoi dialetti. Trad. It. 3 voll. Torino: Einaudi.

Russo, I. (2009). Usi qualificativi degli aggettivi relazionali in italiano e in inglese. Tesi di dottorato. Pisa: Università di Pisa.

Salvi, G. (1981). Complementi predicativi. Studi di grammatica italiana, 10, 313-349.

Serianni, L. (2010 [1989]). Grammatica italiana. Italiano comune e lingua letteraria, con la collaborazione di A. Castelvecchi. Torino: Utet.

SIA = Dardano, M. (a cura di). (2012). Sintassi dell'italiano antico. La prosa del Duecento e del Trecento. Roma: Carocci. 
